\title{
The Yin and Yang of Wnt/Ryk axon guidance in development and regeneration
}

\author{
CLARK Charlotte EJ ${ }^{1}$, LIU Yaobo ${ }^{2} \&$ COOPER Helen $\mathrm{M}^{1 *}$ \\ ${ }^{1}$ Queensland Brain Institute, The University of Queensland, Brisbane, Queensland 4072, Australia; \\ ${ }^{2}$ Institute of Neuroscience, Soochow University, Suzhou 215123, China
}

Received January 17, 2014; accepted February 24, 2014; published online March 14, 2014

\begin{abstract}
In the developing embryo, nascent axons navigate towards their specific targets to establish the intricate network of axonal connections linking neurons within the mature nervous system. Molecular navigational systems comprising repulsive and attractive guidance cues form chemotactic gradients along the pathway of the exploring growth cone. Axon-bound receptors detect these gradients and determine the trajectory of the migrating growth cone. In contrast to their benevolent role in the developing nervous system, repulsive guidance receptors are detrimental to the axon's ability to regenerate after injury in the adult. In this review we explore the essential and beneficial role played by the chemorepulsive Wnt receptor, Ryk/Derailed in axon navigation in the embryonic nervous system (the Yin function). Specifically, we focus on the role of Wnt5a/Rykmediated guidance in the establishment of two major axon tracts in the mammalian central nervous system, the corticospinal tract and the corpus callosum. Recent studies have also identified Ryk as a major suppressor of axonal regeneration after spinal cord injury. Thus, we also discuss this opposing aspect of Ryk function in axonal regeneration where its activity is a major impediment to axon regrowth (the Yang function).
\end{abstract}

Ryk, Derailed, Wnt, Wnt signalling, axon guidance, axonal regeneration, spinal cord injury

Citation: $\quad$ Clark CEJ, Liu YB, Cooper HM. The Yin and Yang of Wnt/Ryk axon guidance in development and regeneration. Sci China Life Sci, 2014, 57: 366-371, doi: 10.1007/s11427-014-4640-3

One of the earliest steps in the development of the central nervous system (CNS) is the initiation of axon outgrowth from newly born neurons. As axons migrate through the complex environments encountered en route to their targets they continually assess the local environment and accurately select the correct pathways among the maze of possible routes. Sophisticated molecular navigational systems comprising secreted or membrane-associated repulsive or attractive guidance cues and their axon-bound receptors govern axon pathfinding. Long-range guidance cues, secreted from intermediate or final targets, form chemotactic gradients along the pathway of the exploring growth cone [1-3].

In contrast to the benevolent role played by guidance molecules in establishing neuronal networks within the developing CNS, repulsive guidance receptor activity is detrimental to the axon's ability to regenerate after injury in the adult CNS. In the case of spinal cord injury, it is now clear that repulsive cues are re-expressed at the injury site, creating a deleterious environment for regeneration [4,5]. Concurrently, the cognate guidance receptors are upregulated on the severed axons, preventing axonal regrowth.

In this review, we focus on the essential role played by Wnt/Ryk signalling in axon navigation in the embryonic brain (the Yin function). We also discuss the opposing aspect of Ryk function in axonal regeneration in the adult, where its activity is a major impediment to axon regrowth (the Yang function).

*Corresponding author (email: h.cooper@uq.edu.au) 


\section{Ryk, a novel Wnt receptor}

$R y k$ was first cloned in the mouse in 1992 as the result of a PCR-based screen for novel receptor tyrosine kinases [6]. However, the function of this unusual receptor and the identity of its ligands remained elusive for more than a decade. In 1999, clues to Ryk's function finally emerged from the fruitfly, Drosophila melanogaster, in which the Ryk orthologue, Derailed, was found to be a chemorepulsive axon guidance receptor [7]. The identity of its ligand was not uncovered until 2003, again as a result of the power of Drosophila genetics. Unexpectedly, the Derailed ligand was identified as Wnt5 [8]. Subsequently, it was demonstrated that Wnt5a/Ryk interactions were required for chemorepulsive axon guidance in the developing mouse brain and spinal cord [9-12].

In vertebrates, more diverse roles are emerging for Ryk in a range of neurodevelopmental processes. Ryk is important for establishing planar polarity during neural tube formation [13-15], differentiation of mouse cortical and dopaminergic midbrain progenitors $[16,17]$, and specification and migration of GABAergic interneurons during cortical development $[18,19]$. Derailed is also required for mushroom body development, glomerular patterning, neuromuscular junction formation, synaptogenesis and memory and learning in the fly [20-23]. These other facets of Ryk function have been comprehensively covered in several recent reviews [24-26].

Ryk/Derailed is an atypical receptor tyrosine kinase due to its inactive tyrosine kinase domain [6,27-29]. Although it is clearly a member of the receptor tyrosine kinase superfamily, there are numerous amino acid substitutions in its kinase domain that render it kinase-dead. Interestingly, although human RYK is also kinase-dead, it is able to activate the MAP kinase pathway [28], possibly via its interaction with the Src kinase family [30]. The Ryk extracellular domain is also unusual as it is considerably shorter than other receptor tyrosine kinases [6]. The predominant features in the extracellular domain are the two leucine-rich motifs, which bear strong homology to the N-terminal domain of Wnt Inhibitory Factor-1 (WIF-1). As predicted, Ryk binds Wnts via these domains $[9,14]$.

Presently, the molecular signalling cascades activated by Wnt/Ryk interactions are not well understood. However, evidence is emerging that Ryk signalling may intersect with both the canonical and non-canonical Wnt pathways depending on the developmental context. Three distinct but interconnected signalling pathways, the canonical $\beta$-catenindependent, the non-canonical Wnt/planar cell polarity (PCP), and the $\mathrm{Wnt} / \mathrm{Ca}^{2+}$ pathways, mediate diverse developmental outcomes triggered by interactions between Wnts and their frizzled $(\mathrm{Fz})$ receptors (reviewed in [24,31,32]). Each pathway is activated by a subset of ligand-receptor pairs, indicating that the biological outcome of Wnt signalling is tightly regulated at the level of ligand-receptor inter- actions. The canonical Wnt pathway, responsible for embryonic patterning and cell fate specification, triggers the nuclear translocation of the transcriptional activator, $\beta$ catenin. In the absence of Wnt, $\beta$-catenin is phosphorylated and targeted to the proteasome for destruction. During axon guidance and cell migration, polarity is established by the PCP pathway, where interactions between a subset of Wnts and Fzs trigger cytoskeletal remodeling [33]. The $\mathrm{Wnt} / \mathrm{Ca}^{2+}$ pathway contributes to some aspects of cell polarity and is closely linked to the PCP pathway. More detailed reviews of Wnt signalling and function in the nervous system can be found in $[24,34,35]$.

Although both canonical (e.g., Wnt1, Wnt3) and noncanonical Wnts (e.g., Wnt5a, Wnt11) have been identified as Ryk ligands [9,13,36], Wnt5a/Ryk interactions have been most intensely studied $[9,11,12,37]$. Axon guidance is dependent on establishing correct polarity within the growth cone by the assembly and disassembly of the actin cytoskeleton. Therefore, one might predict that Ryk would signal through the non-canonical Wnt pathways. Indeed, Wnt5a/Ryk-mediated axon outgrowth and chemorepulsion have been shown to utilise the $\mathrm{Wnt} / \mathrm{Ca}^{2+}$ pathway $[11,37]$. There is also some evidence that Ryk may activate the $\mathrm{Wnt} / \beta$-catenin pathway by forming a co-receptor complex with Fz8 [36] and that Ryk inhibits Wnt/ $\beta$-catenin signalling via interaction with the E3 ubiquitin ligase Mindbomb1 in response to Wnt3a [38]. Currently, our knowledge of the Ryk signalling pathway comes largely from analysis of its axon guidance function.

\section{Lessons from the Drosophila nervous system}

The initial evidence that Ryk was an axon guidance receptor came from the Drosophila Ryk orthologue, Derailed. The Drosophila embryonic nervous system comprises a brain and a ventral nerve cord which consists of two longitudinal axon tracts contacting the brain anteriorly and extending approximately halfway down the longitudinal axis of the embryo [39]. The Drosophila body is divided along the anterior-posterior axis into segments with each segment possessing two commissures, one anterior and one posterior commissure. These facilitate communication between the longitudinal tracts within each body segment. Derailed is expressed by neurons whose axons cross the ventral nerve cord via the anterior commissure (Figure 1). However, these axons do not enter the posterior commissure [7]. Wnt5 is expressed in the posterior commissure and acts as a chemorepulsive ligand preventing Derailed-positive axons from crossing the posterior commissure, instead directing them through the anterior commissure (Figure 1) [8].

As Derailed is a Wnt receptor, it was thought that it may function as a Fz co-receptor to activate the PCP pathway. However, Derailed was found to signal independently of the Fz receptors in the context of Wnt5-dependent chemorepul- 


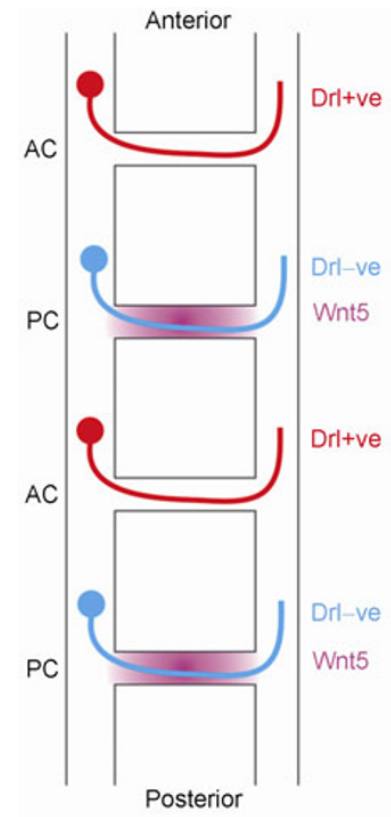

Figure 1 Wnt/Derailed-mediated axon guidance within the Drosophila ventral nerve cord. Derailed-positive axons (Drl+ve, red) cross the midline via the anterior commissure (AC). Wnt5 (purple) is expressed in the posterior commissure $(\mathrm{PC})$, preventing Drl+ve axons from entering this commissure. Derailed-negative axons (Drl-ve, blue) cross via the posterior commissure.

sion [8], providing an early example of a Fz-independent non-canonical Wnt signalling pathway. Evidence to support the contention that Derailed signalled via a non-canonical Wnt pathway has also emerged from experiments using a Derailed-expressing Drosophila S2 cell culture system where addition of Wnt5 failed to activate $\mathrm{Wnt} / \beta$-catenin signalling [30]. The Src non-receptor tyrosine kinases are also required for Wnt5-Derailed-mediated axon guidance. Loss of either Src64B or Src42A leads to disorganised commissures and longitudinal tract defects, a phenotype comparable to that seen in the Wnt5 and Derailed mutants $[30,40]$. Together these studies suggest that Derailed signals via a novel Src kinase-dependent, non-canonical Wnt pathway, at least in the context of chemorepulsive guidance. It is yet to be established whether mammalian Ryk also activates this pathway.

\section{Ryk - an axon guidance receptor in the de- veloping mammalian nervous system}

\subsection{Ryk and the descending corticospinal tract}

The corticospinal tract (CST) comprises axons originating from the pyramidal corticospinal projection neurons in layer 5 of the neocortex and projects subcortically via the internal capsule and then posteriorly within the midbrain. The CST crosses the midline at the pyramidal decussation in the ventral midbrain before projecting through the hindbrain and then along the dorsal funiculus of the spinal cord. Guidance of CST axons begins embryonically with axons first entering the spinal cord at postnatal day 0 (P0) in the mouse, with the remainder of CST axon extension occurring postnatally.

Ryk-dependent chemorepulsion is required for the posterior-directed migration of CST axons down the postnatal spinal cord in response to Wnt gradients (Figure 2) [12]. Ryk is localised to layer 5 neurons at P0 and on CST axons at P5, whereas WntI and Wnt5a are expressed along the trajectory of the descending CST axons within the spinal cord in an anterior-high to posterior-low gradient [12]. In vitro explant assays demonstrated that both Wnt1 and Wnt5a were able to repel P0 CST axons and that anti-Ryk antibodies blocked this effect. Similar results were observed in vivo where anti-Ryk antibodies blocked the posterior pathfinding of CST axons along the dorsal funiculus [12]. This study demonstrated for the first time that Wnt/Rykmediated chemorepulsive guidance was a major axon guidance mechansim in mammals. As seen in Drosophila commissural axon guidance [8], Wnt/Ryk-dependent posterior-directed repulsion down the spinal cord occurred independently of Fzs.

\subsection{Ryk-mediated axon guidance in the mouse corpus callosum}

Within the mammalian forebrain, the corpus callosum is the largest interhemispheric commissure, responsible for information transfer between the two cerebral hemispheres. The

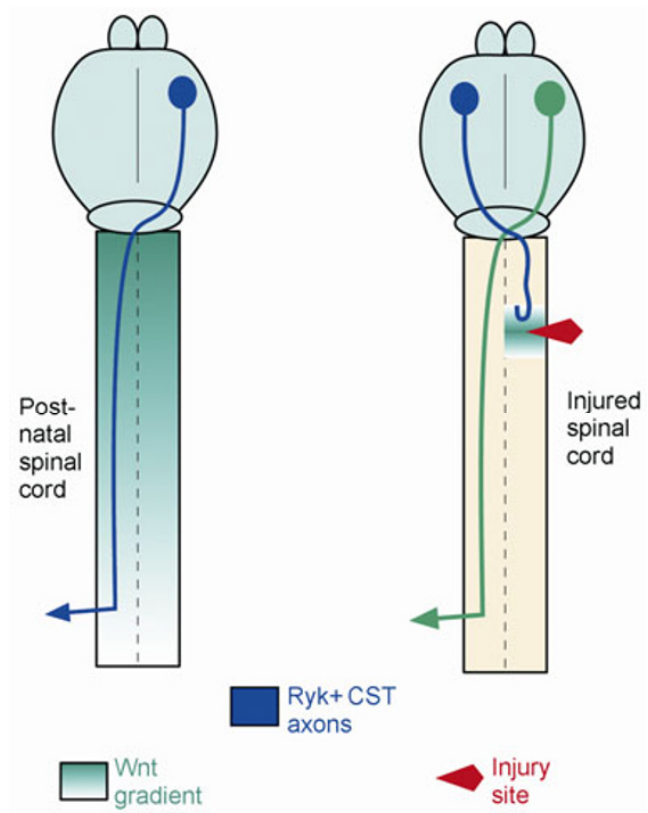

Figure 2 Ryk-dependent chemorepulsion is required for the posteriordirected migration of CST axons down the postnatal spinal cord in response to Wnt1 and Wnt5a gradients. After hemisection of the adult spinal cord, Wnt1 and Wnt5a are upregulated at the injury site and Ryk is re-expressed on injured axons, leading to inhibition of axonal regeneration. 
majority of axons in the corpus callosum derive from neurons located within cortical layers $2 / 3$ and 5 . The pioneer axons of the corpus callosum originating in the cingulate cortex are the first to cross the midline by embryonic day 15 (E15) in the mouse. Axons derived from the rostrolateral neocortex follow the path of the pioneer axons and begin to cross the midline at E16 [41]. Callosal axons continue to cross the midline well into postnatal life after which they integrate into the contralateral cortex.

Ryk is present on callosal projection neurons and their axons, as they approach and then project across the corpus callosum [9]. In the absence of Ryk, callosal axons successfully cross the midline, but fail to project into the contralateral hemisphere [9] (Figure 3). Thus Ryk-mediated axon guidance is required specifically for postcrossing callosal axon guidance. Wnt $5 a$ is expressed by glial cell populations surrounding the callosal midline and promotes Ryk-mediated chemorepulsion of postcrossing (E18) axons away from the midline [9]. Conversely, Ryk is not required for the guidance of precrossing (E16-E17) axons, but does promote their fasciculation [9]. This interpretation was verified by in vitro cortical explant assays showing that in contrast to E18 axons, wildtype E16 and E17 axons were unresponsive to Wnt5a [9]. Together these observations revealed a crucial switch mechanism whereby unresponsive precrossing callosal axons become responsive to $\mathrm{Wnt5a}$ chemorepulsion only after crossing the midline.

Retinotectal mapping of retinal ganglion cell (RGC) axons onto the lateral domain of the chick tectum is also driven by Wnt/Ryk interactions [10]. Ryk is localised on RGC axons in a ventral-high to dorsal-low gradient across the retina. Upon reaching the tectum, interstitial branches projecting from ventral RGC axons are repelled in a Ryk-dependent manner from the Wnt3-high tectal domain, whereas Ryk activity is silenced on dorsal RGC axonal branches in the Wnt3-low lateral tectum. Therefore, tight spatiotemporal regulation of Ryk chemorepulsion is emerging as a common feature throughout vertebrate CNS development.

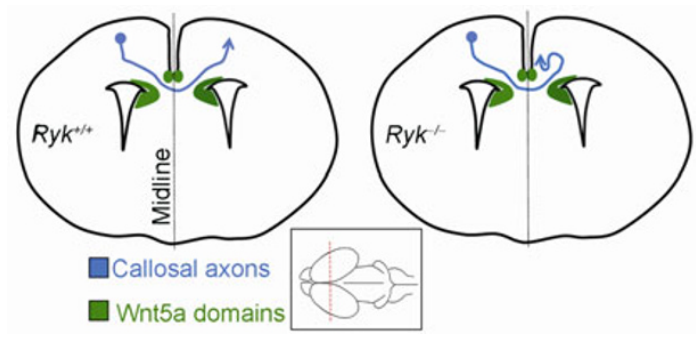

Figure 3 Loss of Ryk leads to misguided contralateral callosal axon guidance. In $R y k^{+/+}$embryos, $R y k$-expressing callosal axons approach and cross the midline, then project into the contralateral hemisphere to make homotopic and heterotopic interhemispheric connections. In $R y k^{-/}$embryos, callosal axons successfully approach and cross the midline, but are unable to project into the contralateral hemisphere. Schematics represent coronal sections through the E18 mouse forebrain.

\section{Spatiotemporal regulation of Ryk chemo- repulsion by the $\mathrm{Wnt5a} / \mathrm{Ca}^{2+}$ pathway}

One unexpected observation in both the CST and corpus callosum was that despite Ryk's chemorepulsive activity, axons continued to grow through high levels of the Wnt chemorepellent $[9,12]$. The solution to this puzzle came from the study of callosal axon guidance in P2 hamster sensorimotor cortex. This study revealed Wnt5a activated two distinct but interconnected arms of the $\mathrm{Wnt} / \mathrm{Ca}^{2+}$ pathway. Axon growth was found to result from Ryk activation of the transient receptor potential (TRP) channels on the plasma membrane and inositol triphosphate (IP3)-mediated $\mathrm{Ca}^{2+}$ release from intracellular stores [11,37]. Conversely, Wnt5a/Ryk-dependent chemorepulsive guidance was shown to require an unidentified $\mathrm{Fz}$ and extracellular $\mathrm{Ca}^{2+}$ entry through TRP channels. However, IP3-mediated $\mathrm{Ca}^{2+}$ release was not involved. In summary, Wnt/Ryk signalling is primarily responsible for axon outgrowth, whereas Ryk and Fz work together to promote chemorepulsive axon guidance. Moreover, pathway specificity is determined downstream by the involvement of IP3-mediated release of intracellular $\mathrm{Ca}^{2+}$. Therefore, Ryk-dependent outgrowth and Ryk/Fzdependent guidance act through distinct arms of the Wnt/ $\mathrm{Ca}^{2+}$ pathway to coordinate axon growth and navigation at key decision points in developing axon tracts.

\section{Silencing Wnt/Ryk interactions enhances axonal regeneration}

Recent studies have identified Ryk as a major suppressor of axonal regeneration after spinal cord injury [42-45]. Based on their initial identification of Ryk as a key guidance receptor in the migration of CST axons along the spinal cord [12], Liu and colleagues hypothesised that Ryk may play an inhibitory role in CST regeneration. A survey of Wnt and Wnt receptor expression after unilateral hemisection of the mouse spinal cord revealed that of the 19 Wnts, only Wnt4, and the Ryk ligands, Wntl and Wnt5a, were acutely upregulated at the injury site, whereas $R y k$ was re-expressed on the lesioned CST axons (Figure 2) [44]. Notably, injection of Ryk inhibitory antibodies into the site of injury prevented CST axon retraction and permitted axonal sprouting. Enhanced sprouting of collateral branches extending into and around the injury site was also observed. A subsequent study has confirmed the effectiveness of Ryk inhibitory antibodies in the promotion of axonal sprouting and further demonstrated enhanced functional recovery as quantified by the Basso-Beatie-Bresnahan locomotor rating scale [45].

A recent follow-up investigation by the Zou lab using a conditioning lesion paradigm [42] has confirmed the predominance of Ryk as an inhibitor of axonal regrowth and has provided further evidence that suppression of Ryk ac- 
tivity may be a viable therapeutic strategy. In this study, Ryk was found to be acutely upregulated after lesioning of the central branches of dorsal root ganglion neurons. Transplantation of bone marrow stromal cells engineered to produce the Wnt inhibitors, WIF1 or SFRP2, at the site of injury promoted regrowth of the ascending sensory axons [42].

In summary, Wnt/Ryk interactions impede regeneration by promoting axonal retraction, and importantly, this detrimental activity can be overcome by the application of reagents that prevent Wnts from binding the Ryk extracellular domain.

\section{Conclusion}

The Wnt/Ryk signalling pathway plays an essential and beneficial role in the establishment of major axon tracts in the developing nervous system across evolutionarily distant phyla [24]. It must be kept in mind, however, that growing axons encounter a complex array of guidance cues within their local environment, including attractive cues such as netrins and other repulsive cues such as the slits [1-3]. Therefore, as multiple receptor activation events impinge on the growth cone at any given point in time and space, the integration of guidance receptor signal transduction is necessary to achieve a synchronous response to the extracellular environment. Non-canonical Wnt/Ryk signalling via the $\mathrm{Wnt} / \mathrm{Ca}^{2+}$ pathway must act in parallel with these diverse guidance systems to ensure error-free axon pathfinding. Presently we have only a poor understanding of how Ryk signalling is integrated with that of other guidance receptor families, including the Fzs, the alternative Wnt receptors. Unraveling these complex signalling networks will greatly expand our understanding of the basic molecular mechanisms underpinning axon guidance in the embryo. Crucially, delineating the Ryk signalling pathway governing embryonic axon guidance may suggest novel strategies with which to enhance repair after spinal cord injury. Ryk is unique among guidance receptors as it has no other family members and so is an ideal therapeutic target.

Charlotte EJ Clark was supported by an Australian Postgraduate Award. We thank the Queensland Government's National and International Research Alliances Program and the National Health and Medical Research Council of Australia for financial support (456040, 569638, 1010723). We also thank the National Natural Science Foundation of China (81330026, 31271259, 30990261, 30871425), the National Basic Research Program, Ministry of Science and Technology of China (2013CB945604), and the Chinese Academy of Sciences (KSCX2-EW-Q-11) for funding support.

1 Dickson BJ, Zou Y. Navigating intermediate targets: the nervous system midline. CSH Persp Biol, 2010, 2: a002055

2 Bashaw GJ, Klein R. Signalling from axon guidance receptors. CSH Persp Biol, 2010, 2: a001941

3 Kolodkin AL, Tessier-Lavigne M. Mechanisms and molecules of neuronal wiring: a primer. CSH Persp Biol, 2010, 3: a001727

4 Giger RJ, Hollis ER, Tuszynski MH. Guidance molecules in axon regeneration. CSH Persp Biol, 2010, 2: a001867

5 Harel NY, Strittmatter SM. Can regenerating axons recapitulate developmental guidance during recovery from spinal cord injury? Nat Rev Neurosci, 2006, 7: 603-616

6 Hovens CM, Stacker SA, Andres AC, Harpur AG, Zimiecki A, Wilks AF. RYK, a receptor tyrosine kinase-related molecule with unusual kinase domain motifs. Proc Nat Acad Sci USA, 1992, 89: 11818-11822

7 Bonkowsky JL, Yoshikawa S, O'Keefe DD, Scully AL, Thomas JB. Axon routing across the midline controlled by the Drosophila Derailed receptor. Nature, 1999, 402: 540-544

8 Yoshikawa S, McKinnon RD, Kokel M, Thomas JB. Wnt-mediated axon guidance via the Drosophila Derailed receptor. Nature, 2003, 422: $583-588$

9 Keeble TR, Halford MM, Seaman C, Kee N, Macheda M, Anderson $\mathrm{RB}$, Stacker SS, Cooper HM. The Wnt receptor Ryk is required for Wnt5a-mediated axon guidance on the contralateral side of the corpus callosum. J Neurosci, 2006, 26: 5840-5848

10 Schmitt AM, Shi J, Wolf AM, Lu CC, King LA, Zou Y. Wnt-Ryk signalling mediates medial-lateral retinotectal topographic mapping. Nature, 2006, 439: 31-37

11 Li L, Hutchins BI, Kalil K. Wnt5a induces simultaneous cortical axon outgrowth and repulsive axon guidance through distinct signalling mechanisms. J Neurosci, 2009, 29: 5873-5883

12 Liu Y, Shi J, Lu CC, Wang ZB, Lyuksyutova AI, Song X, Zou Y. Ryk-mediated Wnt repulsion regulates posterior-directed growth of corticospinal tract. Nat Neurosci, 2005, 8: 1151-1159

13 Kim GH, Her JH, Han JK. Ryk cooperates with Frizzled 7 to promote Wnt11-mediated endocytosis and is essential for Xenopus laevis convergent extension movements. J Cell Biol, 2008, 182: 1073-1082

14 Macheda ML, Sun WW, Kugathasan K, Hogan BM, Bower NI, Halford MM, Zhang YF, Jacques BE, Lieschke GJ, Dabdoub A, Stacker SA. The Wnt receptor Ryk plays a role in mammalian planar cell polarity signalling. J Biol Chem, 2012, 287: 29312-29323

15 Andre P, Wang Q, Wang N, Gao B, Schilit A, Halford MM, Stacker MM, Zhang X, Yang Y. The Wnt coreceptor Ryk regulates Wnt/planar cell polarity by modulating the degradation of the core planar cell polarity component Vang12. J Biol Chem, 2012, 287: 44518-44525

16 Lyu J, Yamamoto V, Lu W. Cleavage of the Wnt receptor Ryk regulates neuronal differentiation during cortical neurogenesis. Dev Cell, 2008, 15: 773-780

17 Blakely BD, Bye CR, Fernando CV, Prasad AA, Pasterkamp J, Macheda ML, Stacker SS, Parish CL. Ryk, a receptor regulating Wnt5a-mediated neurogenesis and axon morphogenesis of ventral midbrain dopaminergic neurons. Stem Cells Dev, 2013, 22: 2132-2144

18 Zhong J, Kim HT, Lyu J, Yoshikawa K, Nakafuku M, Lu W. The Wnt receptor Ryk controls specification of GABAergic neurons versus oligodendrocytes during telencephalon development. Development, 2011, 138: 409-419

19 Kamitori K, Tanaka M, Okuno-Hirasawa T, Kohsaka S. Receptor related to tyrosine kinase RYK regulates cell migration during cortical development. Biochem Biophys Res Comm, 2005, 330: 446-453

20 Dura JM, Taillebourg E, Préat T. The Drosophila learning and memory gene linotte encodes a putative receptor tyrosine kinase homologous to the human RYK gene product. FEBS Lett, 1995, 370: 250-254

21 Liebl FLW, Wu Y, Featherstone DE, Noordermeer JN, Fradkin L, Hing H. Derailed regulates development of the Drosophila neuromuscular junction. Dev Neurobiol, 2008, 68: 152-165

22 Grillenzoni N, Flandre A, Lasbleiz C, Dura JM. Respective roles of the DRL receptor and its ligand WNT5 in Drosophila mushroom body development. Development, 2007, 134: 3089-3097

23 Yao Y, Wu Y, Yin C, Ozawa R, Aigaki T, Wouda RR, Noordermeer JN, Fradkin LG, Hing H. Antagonistic roles of Wnt5 and the Drl 
receptor in patterning the Drosophila antennal lobe. Nat Neurosci, 2007, 10: 1423-1432

24 Clark CEJ, Nourse CC, Cooper HM. The tangled web of non-canonical Wnt signalling in neural migration. NeuroSignals, 2012, 20: 202-220

25 Keeble TR, Cooper HM. Ryk: a novel Wnt receptor regulating axon pathfinding. Int J Biochem Cell Biol, 2006, 38: 2011-2017

26 Fradkin LG, Dura JM, Noordermeer JN. Ryks: new partners for Wnts in the developing and regenerating nervous system. Trends Neurosci, 2010, 33: 84-92

27 Halford MM, Oates AC, Hibbs ML, Wilks AF, Stacker SS. Genomic structure and expression of the mouse growth factor receptor related to tyrosine kinases (Ryk). J Biol Chem, 1999, 274: 7379-7390

28 Katso RM, Russell RB, Ganesan TS. Functional analysis of H-Ryk, an atypical member of the receptor tyrosine kinase family. Mol Cell Biol, 1999, 19: 6427-6440

29 Yoshikawa S, Bonkowsky J, Kokel M, Shyn S, Thomas JB. The derailed guidance receptor does not require kinase activity in vivo. $\mathrm{J}$ Neurosci, 2001, 21: RC119

30 Wouda RR, Bansraj MRKS, de Jong AWM, Noordermeer JN, Fradkin LG. Src family kinases are required for WNT5 signalling through the Derailed/RYK receptor in the Drosophila embryonic central nervous system. Development, 2008, 135: 2277-2287

31 Angers S, Moon RT. Proximal events in Wnt signal transduction. Nat Rev Mol Cell Biol, 2009, 10: 468-477

32 Niehrs C. The complex world of WNT receptor signalling. Nat Rev Mol Cell Biol, 2012, 13: 767-779

33 Shafer B, Onishi K, Lo C, Colakoglu G, Zou Y. Vangl2 promotes Wnt/planar cell polarity-like signalling by antagonizing Dvl1mediated feedback inhibition in growth cone guidance. Dev Cell, 2011, 20: 177-191

34 Harrison-Uy SJ. Wnt signalling and forebrain development. CSH Persp Biol, 2012, 4: a008094

35 Salinas PC, Zou Y. Wnt signalling in neural circuit assembly. Ann
Rev Neurosci, 2008, 31: 339-358

$36 \mathrm{Lu} \mathrm{W}$, Yamamoto V, Ortega B, Baltimore D. Mammalian Ryk is a Wnt coreceptor required for stimulation of neurite outgrowth. Cell, 2004, 119: 97-108

37 Hutchins BI, Li L, Kalil K. Wnt/calcium signalling mediates axon growth and guidance in the developing corpus callosum. Dev Neurobiol, 2011, 71: 269-283

38 Berndt JD, Aoyagi A, Yang P, Anastas JN, Tang L, Moon RT. Mindbomb 1, an E3 ubiquitin ligase, forms a complex with RYK to activate Wnt/B-catenin signalling. J Cell Biol, 2011, 194: 737-750

39 Sánchez-Soriano N, Tear G, Whitington P, Prokop A. Drosophila as a genetic and cellular model for studies on axonal growth. Neural Dev, 2007, 2: 9

40 Petrova IM, Lahaye LL, Martiáñez T, de Jong AWM, Malessy MJ, Verhaagen J, Noordermeer JN, Fradkin LG. Homodimerization of the Wnt receptor DERAILED recruits the Src family kinase SRC64B. Mol Cell Biol, 2013, doi:10.1128/MCB.00169-13

41 Paul LK, Brown WS, Adolphs R, Tyszka JM, Richards LJ, Mukherjee P, Sherr EH. Agenesis of the corpus callosum: genetic, developmental and functional aspects of connectivity. Nat Rev Neurosci, 2007, 8: 287-299

42 Hollis ER, Zou Y. Reinduced Wnt signalling limits regenerative potential of sensory axons in the spinal cord following conditioning lesion. Proc Nat Acad Sci USA, 2012, 109: 14663-14668

$43 \mathrm{Li} \mathrm{X}, \mathrm{Li} \mathrm{YH}, \mathrm{Yu}$ S, Liu Y. Upregulation of Ryk expression in rat dorsal root ganglia after peripheral nerve injury. Brain Res Bull, 2008, 77: $178-184$

44 Liu Y, Wang X, Lu CC, Kerman R, Steward O, Xu XM, Zou Y. Repulsive Wnt signalling inhibits axon regeneration after CNS injury. J Neurosci, 2008, 28: 8376-8382

45 Miyashita T, Koda M, Kitajo K, Yamazaki M, Takahashi K, Kikuchi A, Yamashita T. Wnt-Ryk signalling mediates axon growth inhibition and limits functional recovery after spinal cord injury. J Neurotrauma, 2009, 26: 955-964

Open Access This article is distributed under the terms of the Creative Commons Attribution License which permits any use, distribution, and reproduction in any medium, provided the original author(s) and source are credited. 